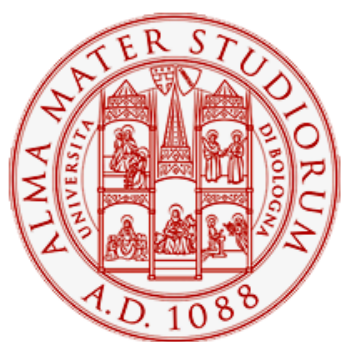

Alma Mater Studiorum - Università di Bologna DEPARTMENT OF ECONOMICS

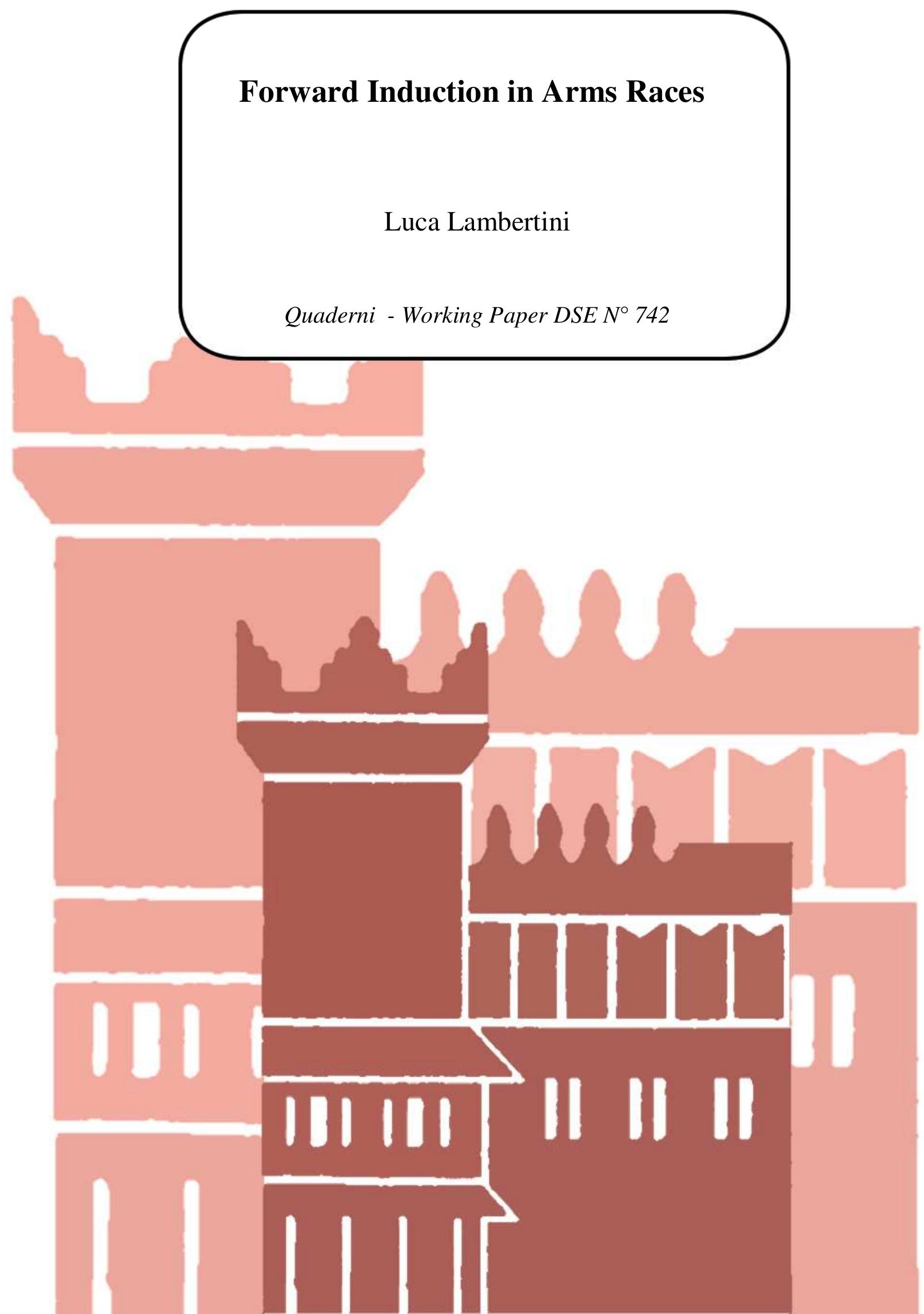




\title{
Forward Induction in Arms Races
}

\author{
Luca Lambertini \\ Department of Economics, University of Bologna \\ Strada Maggiore 45, 40125 Bologna, Italy \\ luca.lambertini@unibo.it; fax: +39-051-2092664 \\ ENCORE, University of Amsterdam \\ Roetersstraat 11, WB1018 Amsterdam, The Netherlands \\ RCEA, via Angherà 22, 47100 Rimini, Italy \\ February 18, 2011
}

\begin{abstract}
I investigate a two-country non cooperative game where the status quo ante is asymmetric as one country is endowed with nuclear weapons while the other is not and is evaluating the opportunity of build up a nuclear arsenal. After identifying the conditions on payoffs such that the resulting reduced form is a coordination game with two symmetric equilibria, I resort to forward induction to show that the implicit signalling mechanism in it may lead countries to select the peaceful equilibrium in a symmetric environment where both are endowed with analogous arsenals.
\end{abstract}




\section{Introduction}

May arms races have destabilising effects, and ultimately bring about the outbreak of war? This question has been - and still is - intensively debated in the literature (see, e.g., Brito and Intriligator, 1984; Powell, 1987; Chassang and Padró I Miquel, 2009). A related crucial aspect is the effectiveness of nuclear deterrence in preventing this outcome, whereby weapons are being built precisely in order to prevent a war (see Powell, 1990; Wagner, 1991; Zagare, 2004, inter alia). Consequently, while in line of principle there can be a generalised agreement on the fact that a world without weapons is trivially preferable to one where each country avails of a non negligible arsenal, the appraisal of the balance between the pros and cons of taking part in an arms race remains somewhat ambiguous.

Additionally, this may impose on countries a disproportionate cost. Ever since Shubik's dollar auction game (Shubik, 1971; see also O'Neill 1986 and Leininger, 1989, inter alia) we know that playing games involving arms races is a very harsh task in economic terms, as countries face the concrete possibility of excess investment, or even default. Consequently, a country may decide never to engage in an arms race, although this involves the unpleasant consequence of being put at a permanent disadvantage against other countries that have instead chosen to undertake such enterprise.

Another way of approaching this matter consists in asking oneself whether a scenario where all countries are endowed with considerable arsenals (typically including nuclear weapons) is more or less conducive to open conflicts as compared to alternative situations where such weapons are totally absent or very unevenly distributed across countries. Furthermore, is it correct to 
interpret a country's effort to put itself on equal footing with others already endowed with strategic weapons as a necessarily hostile move? The objective of this note is to examine this problem using the tool of forward induction in an asymmetric game in which, at the outset, one country is a nuclear power while the other is not, but is considering the possibility of acquiring a nuclear arsenal of its own. It turns out that, if the game yields two symmetric Nash equilibria posing a coordination problem that cannot be solved by backward induction, invoking the forward induction argument indeed works as an equilibrium selection tool, in such a way that an even endowment of nuclear weapons drives countries to the peaceful equilibrium.

\section{The game}

To begin with, consider the following non cooperative game. Players are two fully symmetric countries, labelled as 1 and 2. They are both nuclear powers, and the game consists in deciding whether to strike or not. The two pure strategies are $a$ for to attack and na for not to attack. This is a one-shot game taking place under imperfect, complete and symmetric information. The corresponding $2 \times 2$ strategic form is described by Matrix 1 .

2

\begin{tabular}{cc|c|c|}
\multicolumn{1}{c}{} & \multicolumn{1}{c}{$a$} & \multicolumn{1}{c}{$n a$} \\
\cline { 3 - 4 } 1 & $a$ & $w ; w$ & $f ; s$ \\
\cline { 3 - 4 } & $n a$ & $s ; f$ & $p ; p$ \\
\cline { 3 - 4 } & & &
\end{tabular}

Matrix 1 
The payoffs appearing in the cells can be read as $w=$ war, $p=$ peace, $f=$ first and $s$ =second, respectively. Asymmetric outcomes $(a, n a)$ and $(n a, a)$ are to be interpreted as the reduced form of scenarios where one country strikes first but the other is not altogether annihilated and therefore is in a position to retaliate, at least to some extent. ${ }^{1}$ The equilibrium outcomes generated by the game of course depend on the relevant payoff ranking. To begin with, while it makes sense to assume that $p>w$, we may say that if hawks (respectively, doves) are in control, then $f>p$ (respectively, $p>f){ }^{2}$ Therefore, as in Lichbach (1990), we may ask ourselves what kind of game these two countries are about to play. The overall appraisal of Matrix 1 reveals the following:

- if $w>s$, i.e., a symmetric war is better than bearing the brunt of a first strike, and $p>f$, then we have a coordination game along the main diagonal, $(a, a)$ and $(n a, n a)$ being both Nash equilibrium outcomes. It is worth stressing that the inequality $p>f$ is compatible with a forward looking attitude on the part of both players, if they are taking into account the long term global (i.e., economic, climatic, demographic) consequences of any - even limited - use of nuclear weapons; ${ }^{3}$

\footnotetext{
${ }^{1}$ For a similar structure, see Chassang and Padró I Miquel (2009). They focus, however, on the potentially destabilising effects of defensive shields, such as the Strategic Defense Initiative carried out by the US under the Reagan administration.

${ }^{2}$ This aspect is particularly delicate and has ultimately to do with the preferences of those in control of nuclear arsenals. A relevant example, in this respect, is the inclination of General Curtis LeMay to interpret the mission of the Strategic Air Command as that of performing an all-out attack at the very beginning of a war. This strategy was labelled as the "Sunday punch". For more on this, see Rhodes (1995).

${ }^{3}$ On this particular aspect, see Mills et al. (2008), inter alia.
} 
- if $s>w$ and $p>f$, then the game has a unique pure-strategy Nash equilibrium in $(n a, n a)$, which is also Pareto-efficient;

- if $s>w$ and $f>p$, then we observe a chicken game with two purestrategy equilibria along the secondary diagonal, at $(a, n a)$ and $(n a, a)$;

- if $w>s$ and $f>p$, then the game is a prisoners' dilemma yielding a unique and Pareto-inefficient pure-strategy Nash equilibrium in correspondence of $(a, a)$.

Whenever the game yields two pure strategy equilibria, the related coordination problem hinders our capability of predicting the exact outcome of the game; additionally, it prompts for the analysis of the mixed strategy equilibrium and possibly also for the investigation of risk dominance. My objective in the remainder of this note is instead to propose the use of forward induction (see Kohlberg and Mertens, 1986; and van Damme, 1991) for the specific case in which the game poses a coordination problem, the two equilibria being $(a, a)$ and $(n a, n a)$. 


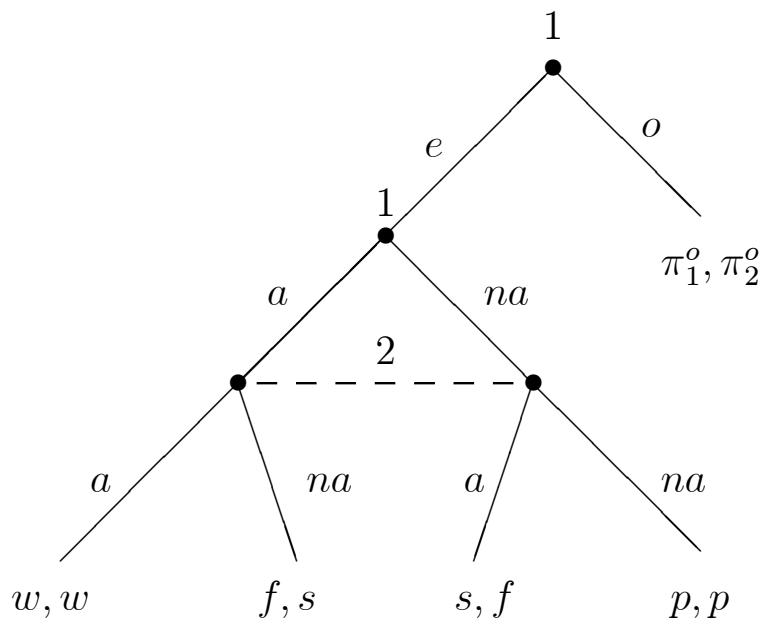

Figure 1 The extensive form with an outside option

To envisage a way out of the coordination issue, we can step back and imagine an alternative situation where the outbreak of a nuclear conflict is not plausible as country 2 is already a nuclear power, while country 1 is not, but may decide to become one. If it does, there indeed arises the perspective of a symmetric nuclear confrontation possibly leading to a war. Otherwise, the status quo ante persists, with country 2 enjoying a dominant position in the international scenario by virtue of its exclusive endowment of nuclear weapons, and may extract a positional rent from this very fact.

Now observe the game tree in Figure 1. Country 1 has the option to stay out of the nuke club (strategy $o$ ). If it does so, the resulting payoffs 
are $\pi_{2}^{o}>\pi_{1}^{o}$, this inequality capturing the aforementioned positional rent. Hence, should it decide to remain out of the club, country 1 would put itself at disadvantage with respect to country 2 , which might then be in a position to impose humiliating decisions onto country 1 . By entering instead the club (strategy $e$ ), country 1 leads both players to a subgame with imperfect information (although complete and symmetric) equivalent to the strategic form described in Matrix 1. Matrix 2 illustrates the strategic form corresponding to the tree.

\begin{tabular}{cc|c|c|}
\multicolumn{1}{c}{} & \multicolumn{2}{c}{$a$} & \multicolumn{1}{c}{$n a$} \\
\cline { 3 - 4 } & $e a$ & $w ; w$ & $f ; s$ \\
\cline { 3 - 4 } 1 & ena & $s ; f$ & $p ; p$ \\
\cline { 3 - 4 } & & $\pi_{1}^{o} ; \pi_{2}^{o}$ & $\pi_{1}^{o} ; \pi_{2}^{o}$ \\
\cline { 2 - 4 } & &
\end{tabular}

Matrix 2

For $(a, a)$ and $(n a, n a)$ to be the Nash equilibria of the imperfect information subgame, it must be true that $w>s$ and $p>f$. Additionally, remember that I have also assumed $p>w$. Assume, further, that $\pi_{1}^{o} \in(\max \{w, f\}, p)$ and $\pi_{2}^{o}>p$, the latter inequality being in agreement with the idea that holding monopoly power on nuclear weapons would put country 2 in a dominant position in the international arena.

The principle of backward induction cannot help solve the imperfect information subgame that generates if country 1 chooses $e$. Instead, forward 
induction can be used to perform this task. According to the forward induction principle, each player - in order to forecast future developments, and ultimately the equilibrium outcome, starting from any node of a sequential play game - has to interpret correctly the reasons why the game has reached that particular node and not others. To do this, every player has to figure out the reasons behind the behaviour of any other players that moved at previous nodes. This also entails that each player uses an implicit signalling mechanism at every node, so as to transmit to others a rational hint about what he/she expects to happen in the remainder of the game.

If indeed $\pi_{1}^{o} \in(\max \{w, f\}, p)$, by playing $e$ country 1 signals that it expects the game to produce the peaceful equilibrium $(n a, n a)$, and country 2 should understand this on the basis of forward induction. The same conclusion can be reached by iterated deletion of weakly dominated strategies in Matrix 2. Examine first the strategic form from the standpoint of country 1: given that $\pi_{1}^{o}>\max \{w, f\}$ and $w>s$, strategy ea is strictly dominated by strategy $o$, so that the second row can be deleted. This is known to country 2 , that, on the resulting $2 \times 2$ matrix finds out that $n a$ weakly dominates $a$ since $p>f$. The last step consists in observing that along the remaining $2 \times 1$ column is such that country 1 has in ena a dominant strategy. Therefore, iterated dominance produces indeed to the same equilibrium outcome as forward induction. 


\section{References}

[1] Brito, D.L. and M.D. Intriligator (1984), "Can Arms Races Lead to the Outbreak of War?", Journal of Conflict Resolution, 28, 63-84.

[2] Chassang, S. and G. Padró I Miquel (2009), "Defensive Weapons and Defensive Alliances", American Economic Review, 99 (P\&P), 282-86.

[3] Kohlberg, E. and J.-F. Mertens (1986), "On the Strategic Stability of Equilibria", Econometrica, 54, 1003-38.

[4] Leininger, W. (1989), "Escalation and Cooperation in Conflict Situations: The Dollar Auction Revisited", Journal of Conflict Resolution, 33, 231-54.

[5] Lichbach, M.I. (1990), "When Is an Arms Rivalry a Prisoner's Dilemma? Richardson's Models and $2 \times 2$ Games", Journal of Conflict Resolution, 34, 29-56.

[6] Mills, M.J., O.B. Toon, R.P. Turco, D.E. Kinnison and R.R. Garcia (2008), "Massive Global Ozone Loss Predicted following Regional Nuclear Conflict", Proceedings of the National Academy of Sciences, 105, 5307-12.

[7] O’Neill, B. (1986), "International Escalation and the Dollar Auction", Journal of Conflict Resolution, 30, 33-50.

[8] Powell, R. (1987), "Crisis Bargaining, Escalation, and MAD", American Political Science Review, 81, 717-36. 
[9] Powell, R. (1990), Nuclear Deterrence Theory. The Search for Credibility, Cambridge, Cambridge University Press.

[10] Rhodes, R. (1995), Dark Sun. The Making of the Hydrogen Bomb, New York, Simon and Schuster.

[11] Shubik, M. (1971), "The Dollar Auction Game: A Paradox in Noncooperative Behavior and Escalation", Journal of Conflict Resolution, 15, 109-11.

[12] van Damme, E. (1991), Stability and Perfection of Nash Equilibria, Heidelberg, Springer-Verlag.

[13] Wagner, R.H. (1991), "Nuclear Deterrence, Counterforce Strategies, and the Incentive to Strike First", American Political Science Review, 85, 727-49.

[14] Zagare, F. (2004), "Reconciling Rationality with Deterrence. A Reexamination of the Logical Foundations of Deterrence Theory", Journal of Theoretical Politics, 16, 107-41. 


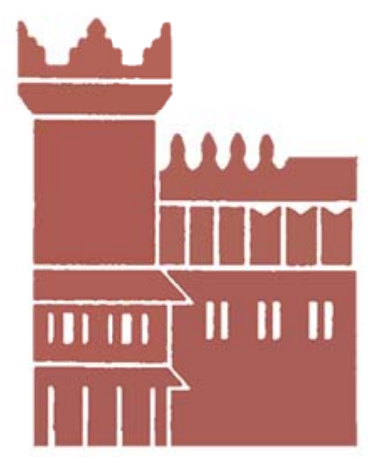

Alma Mater Studiorum - Università di Bologna DEPARTMENT OF ECONOMICS

Strada Maggiore 45

40125 Bologna - Italy

Tel. +39051 2092604

Fax +390512092664

http://www.dse.unibo.it 